\title{
A Simple Procedure for Preparation of $N$-Thiazol, Thiadiazol, Pyridyl and Sulfanylamidocantharidinimines Analogues and Evaluation of Their Cytotoxicities against Human HL-60, MCF7, Neuro-2a and A549 Carcinoma Cell
}

\author{
Ing-Jy Tseng, ${ }^{a}$ Shiow-Yunn Sheu, ${ }^{b}$ Ying-Tzu Chen, ${ }^{c}$ Chu-Yun Huang, ${ }^{c}$ Ching-Tung Lin, ${ }^{e}$ and \\ Pen-Yuan Lin ${ }^{*}, d$ \\ ${ }^{a}$ School of Geriatric Nursing and Care Management, Taipei Medical University; ${ }^{b}$ Department of Pharmaceutical \\ Analysis, School of Pharmacy, Taipei Medical University; ' Department of Clinical Pharmacy, School of Pharmacy, \\ Taipei Medical University; ${ }^{e}$ Department of Pharmaceutical Sciences, School of Pharmacy, Taipei Medical University; \\ 250 Wu-Hsing St., 11031 Taipei, Taiwan: and ${ }^{d}$ Department of Chemistry, Tam-Kang University; 151 Yingzhuan Rd. \\ Danshui Dist, 25137 New Taipei City, Taiwan. Received May 7, 2012; accepted August 3, 2012
}

The lab made an effort to prepare some biological active cantharidinimines by heating the reactant 1 and $2 \mathrm{a}-\mathrm{g}, 5 \mathrm{~h}-\mathbf{i}$ and $7 \mathbf{j}-\mathbf{r}$ amines to suitable temperature with ethanol to provide $18 \mathrm{~N}$-thiazolyl-, sulfanyl-, aminopyridyl-, bromopyridyl-, alkylpyridyl- and hydroxypyridylcantharidinimines $3 \mathbf{a}-\mathrm{g}, 4 \mathrm{a}-\mathrm{c}, 6 \mathrm{~h}-\mathbf{i}$ and $8 \mathbf{j}-\mathbf{r}$ in yield of $4-77 \%$ (Chart 1). These cantharidinimine derivatives were tested for their capabilities to suppress growth of the human carcinoma cell lines, HL-60, MCF7, Neuro-2a and A549, because the incidence rate is more prominent in Asian countries than western countries. Compounds $3 \mathrm{c}-\mathrm{d}$ and $\mathbf{6 h}-\mathbf{i}$ were found to have some antitumor activity in HL-60 but less activity in MCF cell and compounds $8 j-1$ displayed some inhibition effects to A549 cell line, but less effect to Neuro-2a cell line. Compounds 8m-r had no cytotoxic effect against both cell lines. The cytotoxic effects of these cantharidinimine compounds seemed to be better than the cantharidinimide compounds which we had mentioned several years ago.

Key words cantharidin; $N$-azayl-thiadiazolylcantharidinimine; cytotoxicity; sulfanyl-thiadiazolylcantharidinimine; thiazolyl-thiadiazolylcantharidinimine

Cantharidin $\mathbf{1}$ is found in Mylabris caraganae and various other insects and shows extremely high vesicant potency and toxic properties. ${ }^{1-4)}$ Recent reports have indicated that the action of cantharidin in mice is associated with binding to phosphorylase $2 \mathrm{~A}$ in liver cytosol and inhibition of its phosphorylase phosphastase activity. ${ }^{5-9)}$ In our previous studies, ${ }^{10}$ increasing the solubility of the title compounds decreased the toxicity while maintaining the biological activities. $N$-Thiazolyl, sulfanylcantharidinimides with a better solubility showed high cytotoxicity against human carcinoma cell lines. The present study shows that characters of amine basicity and the chosen temperature are crucial. Furthermore the characters of the groups and their position on the aromatic or azalyl ring also influence yields. In order to obtain novel types of related imines and study the scope of these synthetic reactions, the same technique was applied to the reaction of compounds 1 with thiazolyl, thiadiazolyl, sulfanyl, or azalylamines in high-pressure tube with dry ethanol and heated to $c a .140^{\circ} \mathrm{C}$. This temperature and reaction time influence the product yields. The method might give good yields after evaporated and recryatallization in methanol (Table 1). In our previous research, ${ }^{11)}$ we synthesized many cantharidinimide derivatives by modification with aliphatic, azalyl, thiazolyl, thiadiazolyl and pyridyl groups. Those compounds exhibited a certain degree of cytotoxic activity on HL-60, Hep3B and HepG2, ${ }^{3-5)}$ NUGC (Human Gastric carcinoma cells), HONZ-1 (Human Nasopharyngeal carcino cells) ${ }^{11-16)}$. All of these compounds contained an imide group. Thus, we use this feature to synthesize different imine derivatives to study effects of antitumor therapy and cytotoxicity as carcinoma cells inhibitors

The authors declare no conflict of interest. and therapy sources.

\section{Results and Discussion}

With the high-pressure technique, we obtained cantharidinimines (after recrystallization from methanol) in yields of $4-77 \%$, as listed in Table 1 . The highest yields were obtained from dimethylpyridyl $\mathbf{7 q}$ as well as thiazoylamines $\mathbf{2 a - e}$. The $\mathrm{NH}_{2}$ basicities of aminothiazolyl-, aminosulfanyl- and pyridylcantharidinimine are unknown but will be slightly different between one of corresponding aminothiazoyl-, aminosulfanyland pyridylamine. There is an electron deficiency of the thiazol, sulfanylamide, aza and aminopyridine rings. Compounds $\mathbf{2 h}-\mathbf{i}$ which had the sulfanyl and pyridyl ring exerted the most electron withdrawing capability with resonance and induction effects. The formation of cantharidinimine appeared to become more difficult hence showed the very low yields, $6 \mathbf{h}$ and 6i. We have noted in our previous works that the more conjugated character, the higher the yield obtained. Withdrawing functional groups on the aza ring always gave a lower yield than the electron donating group. Compounds $\mathbf{4 a - c}$ were obtained from the reduction of compounds $\mathbf{3 a}-\mathbf{c}$ by using reducted reagent, $\mathrm{NaBH}_{4}$ with ethanol at room temperature and refluxed to give yields $28 \%, 14 \%$ and $2 \%$. Their NMR values displayed the singlet signal at $\delta 5.78,5.80$ and 5.87 for one $\mathrm{H}$ (reducted hydrogen) and IR showed the absorption of reducted of imines and $\mathrm{C}=\mathrm{O}$ at $1700,3492 \mathrm{~cm}^{-1}$ for $\mathbf{4 a}$, at 1701 , $3440 \mathrm{~cm}^{-1}$ for $\mathbf{4 b}$ and at $1692,3417 \mathrm{~cm}^{-1}$ for $\mathbf{4 c}$, and all of their mass spectra were obtained on a Joel JMX-DX300 for high resolution spectrometer. The variable yields of compounds $\mathbf{2 d}-\mathbf{g}, \mathbf{2 h}-\mathbf{i}, \mathbf{8 j}-\mathbf{r}$, which had parent aryl, azalyl, sulfanyl and pyridyl compounds might reflect the inductive electron negative, bulkyl steric, or the anhydride oxygen linkage electrical 

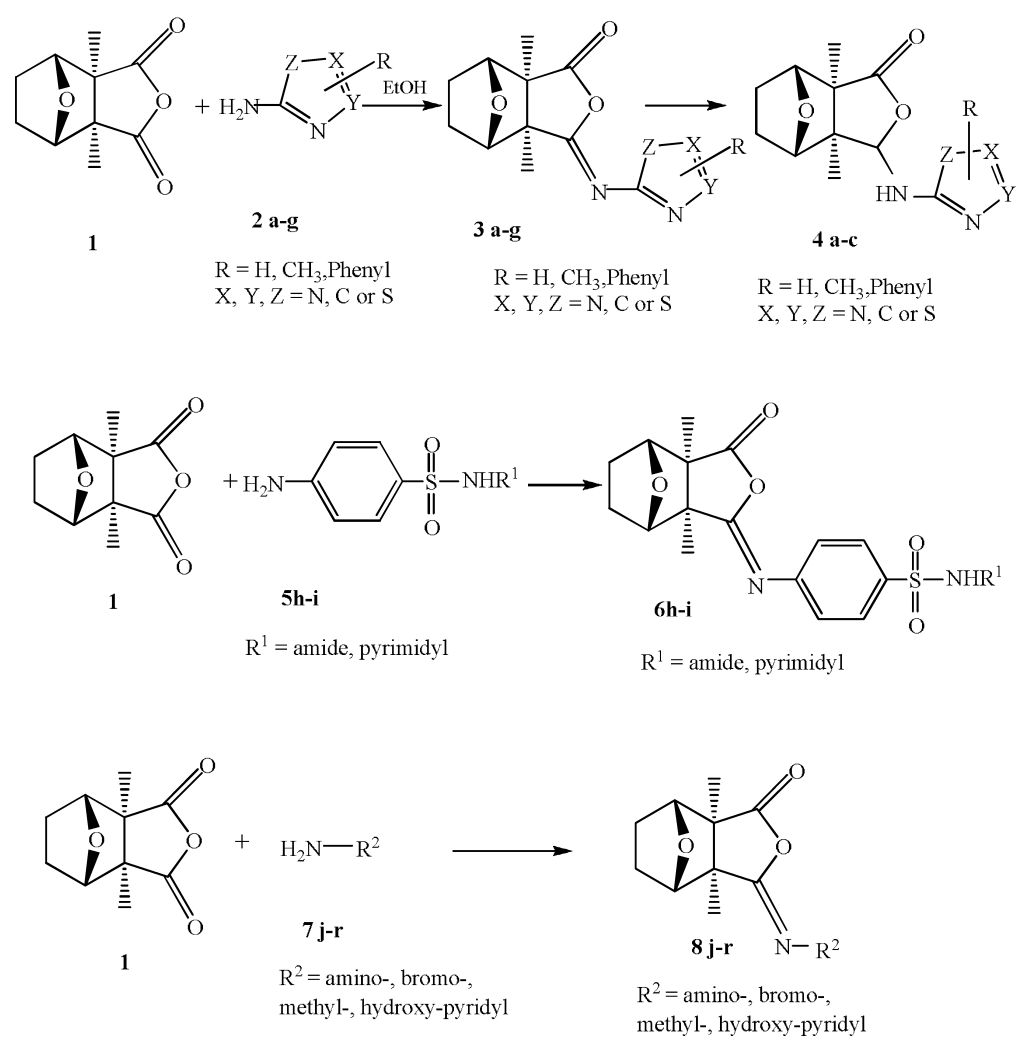

Chart 1

density effect influencing the yields.

The results of these yields strongly confirm the influence of amine nucleophilicity and basicity. The potential cytotoxicities of prepared cantharidinimines were investigated against human myeloid leukemia cell (HL-60), human breast adenocarcinoma (MCF7, from ATCC), rat brain neuroblastoma (Neuro-2a, $\mathrm{N}_{2} \mathrm{a}$, from ATCC) and human lung carcinoma (A549, from ATCC) lines and valuated. Using 3-(4,5-dimethylthiazol-2-yl)-2,5-diphenyltetrazolium bromide (MTT) cell viability assay (Table 2) for cytotoxicity tests to show cantharidin and its cantharidinimine analogues listed in Table $1, \mathbf{3 a}-\mathbf{g}, \mathbf{6 h}-\mathbf{i}, \mathbf{8 j}-\mathbf{r}$. Cantharidin $\mathbf{1}$ was more toxic and exhibited greater cytotoxicity. While synthesized cantharidinimines were less toxic but also exhibited inhibitory effects. Being comparable in cytotoxicity to cantharidin, the $\mathrm{IC}_{50}$ values of all of the cantharidinimine derivatives $\mathbf{3 a}-\mathbf{8 r}$ were 6.0 to $100 \mu \mathrm{M}$, and of cantharidin were $4.6-82.8 \mu \mathrm{M}$ and of $\mathbf{3 a}-\mathbf{c}$ and $\mathbf{3 g}$ were completely inactive up to the high concentration tested $(100 \mu \mathrm{M})$. Compounds $\mathbf{3 d}-\mathbf{f}$ have thiadiazolyl functional group showed a higher cytotoxicity than $\mathbf{3 a}-\mathbf{c}$ and $\mathbf{3 g}$. Compounds $\mathbf{3 d}$ and $\mathbf{3 f}$ had azoyl ring structure that gave the same cytotoxicities. Sulfanylamine derivatives with two methyl substitutes, $\mathbf{6 h}-\mathbf{i}$. showed high cytotoxicities to HL-60 cell, $\mathbf{6 h}$ displayed more cytotoxicity than 6i. with a different position of pyridyl ring. Unfortunately they all showed inactivities up to the MCF7 cell line. Compounds $\mathbf{8 j} \mathbf{j}-\mathbf{r}$ had amino substitutes and pyridyl group which might show that the presence of electron donating substitute, $\mathbf{8 1}, \mathbf{8 m}, \mathbf{8 n}$ and $\mathbf{8 r}$ enhanced cytotoxicities remarkably. Compounds $\mathbf{8 j} \mathbf{-}-\mathbf{l}$ showed cytotoxic activity to A549 cell line but displayed no activity toward Neuro-2a cell line. It might have some effects for other cell lines and the less cytotoxicity might be used to become as prodrug references. Compared to our previous works, we noticed that these cantharidinimine derivatives had more cytotoxic effects than cantharidinimides, especially in the presence of sulfanylamide groups, and the diamino derivatives of cantharidinimine also showed the activities better than its cantharidinimide derivatives. We will continue this works in the future.

\section{Experimental}

Test Samples Chinese blister beetles were extracted with a water-ethanol ratio of $1: 1$ solution, filtered with celite, purified by chromatography on silica gel, and then recrystallized with ethanol to give cantharidin 1. Compound 3-8 were prepared from cantharidin and primary amines in the presence of triethylamine in ethanol in a high-pressure tube. IR spectra were recorded on a Thermo Mattson IR300 spectrometer. NMR spectra were recorded on a Bruker DRX-500 spectrometer in pyridin- $d_{5}$ solution with respect to the corresponding solvent as the internal standard. ${ }^{1} \mathrm{H}$ - and ${ }^{13} \mathrm{C}-\mathrm{NMR}$ spectra were measured at 500 and $125 \mathrm{MHz}$, respectively. NMR experiments included ${ }^{1} \mathrm{H}-{ }^{1} \mathrm{H}$ correlation spectroscopy (COSY), distortionless enchancement by polarization transfer (DEPT), ${ }^{1} \mathrm{H}$ detected heteronuclear multiple quantum coherence (HMQC) and heteronuclear multiple bond connectivity (HMBC). All chemical shifts were expressed in ppm $(\delta)$. Multiplicities of all carbon signals were verified through DEPT experiments. Mass spectra were measured on a Finnigan/Thermo Quest MAT 95XL, JEOL JMX-HX 110 and JEOL JMX-DX 300 for high or low resolution spectrometers. ${ }^{14)}$ For the chromatographic analysis Merck Silica Gel 60 (230-400 mesh ASTM) was used. The chemical reagents used in synthesis were purchased 
Table 1. Preparation of Cantharidinimines
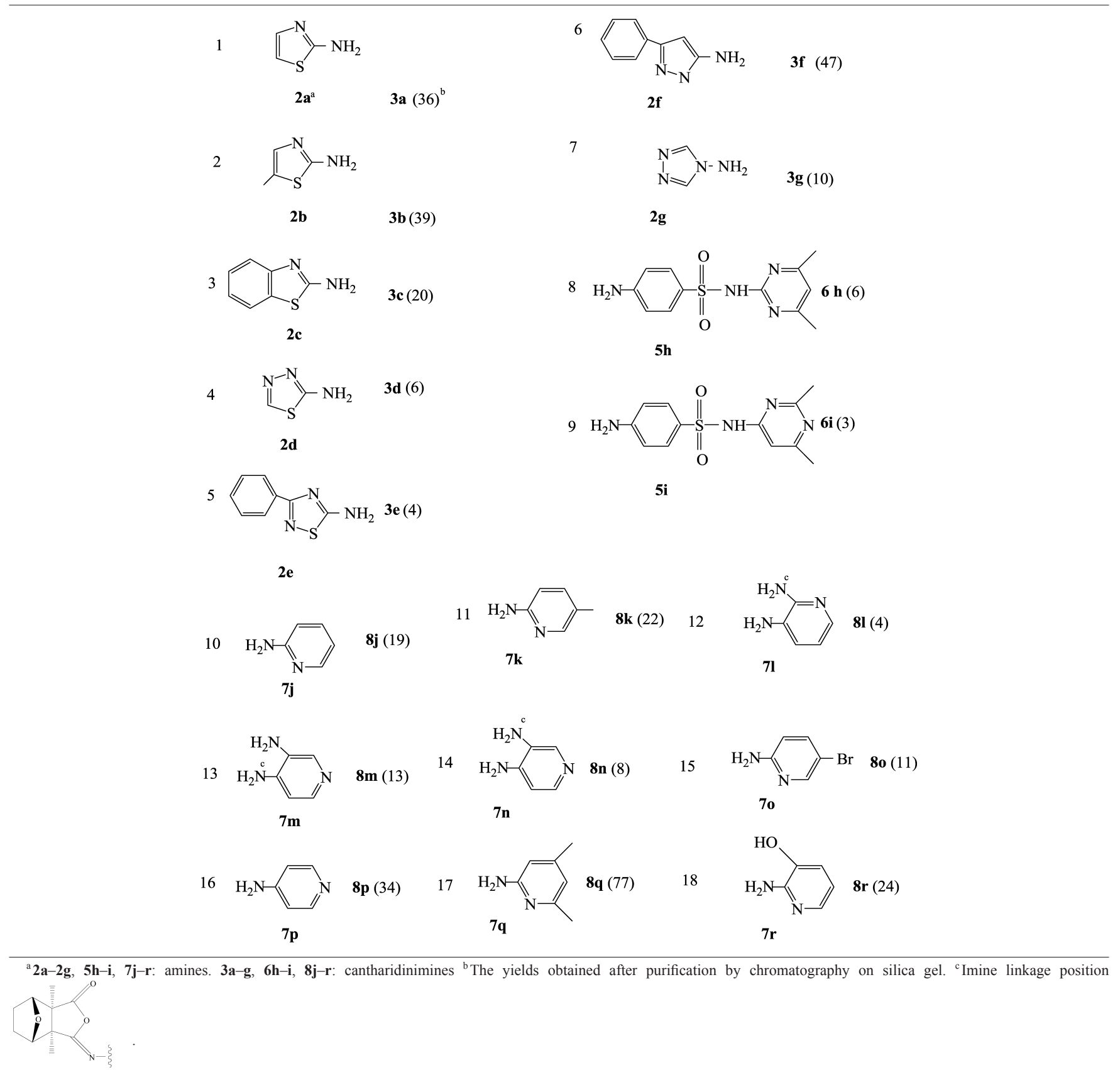

Cantharidinimine

Table 2. Cytotoxicity of Cantharidin 1, N-Thiazol-, $N$-Thiadiazol-, and Sulfanylcantharidinimines in Human HL-60, MCF7, Nuro-2a, A549 Carcinoma Cells

\begin{tabular}{lccccccccccc}
\hline \hline \multicolumn{10}{c}{} & \multicolumn{10}{c}{$\mathrm{IC}_{50}(\mu \mathrm{M})^{a)}$} \\
\hline Cell line & $\mathbf{1}$ & $\mathbf{3 a}$ & $\mathbf{3 b}$ & $\mathbf{3 c}$ & $\mathbf{3 d}$ & $\mathbf{3 e}$ & $\mathbf{3 f}$ & $\mathbf{3 g}$ & $\mathbf{6 h}$ & $\mathbf{6 i}$ \\
\hline HL-60 & 7.2 & $>100^{b)}$ & $>100$ & $>100$ & 17.7 & 41.9 & 18.1 & $>100$ & 6 & 35.9 \\
MCF7 & 82.8 & $>100$ & $>100$ & $>100$ & $>100$ & $>100$ & $>100$ & 100 & $>100$ & $>100$ \\
\hline & $\mathbf{1}$ & $\mathbf{8 j}$ & $\mathbf{8 k}$ & $\mathbf{8 1}$ & $\mathbf{8 m}$ & $\mathbf{8 n}$ & $\mathbf{8 0}$ & $\mathbf{8 p}$ & $\mathbf{8 q}$ & $\mathbf{8 r}$ \\
\hline Nuro-2a & 70.4 & $>100$ & $>100$ & $>100$ & $>100$ & $>100$ & $>100$ & $>100$ & $>100$ & $>100$ \\
A549 & 4.57 & 21.6 & 21.1 & 31.8 & $>100$ & $>100$ & $>100$ & $>100$ & $>100$ & $>100$ \\
\hline
\end{tabular}

a) $\mathrm{IC}_{50}$ was calculated after $48 \mathrm{~h}$ of continuous drug exposure, Values are the mean of three to four experiments with coefficients of variation of $5-10 \%$. $b$ ) No indication of the cytotoxicity was considered indication of $\mathrm{IC}_{50}$ values substantially rather than the highest doses assayed. 
from Sigma-Aldrich Co. and E. Merck.

General Procedures Genaeral procedures were followed for the reaction of primary amines with cantharidin. These compounds were prepared according to similar procedures, and the reaction took place in high-pressure tubes. Cantharidin was added to a tube containing $3 \mathrm{~mL}$ of dry ethanol and triethylamine, and the solution was stirred and heated to $c a$. $200^{\circ} \mathrm{C}$. After being stirred for $2 \mathrm{~h}$, the mixture was evaporated, and the residue mass was purified by column chromatography and recrystallized from methanol.

\section{Antineoplastic Bioassays}

Preparation of Test Solution and Assay Procedure for Cytotoxicity Media and sera for cell culture were purchased from Gibco/BRI (Grand Island, NY). Most chemicals were purchased from Sigma Chemical Co. (St. Louis, MO, U.S.A.). HL-60 (Human promyelocytic leukemia), MRC-5 (human hepatocellular H460 (non-small cell lung cancer tumor cell) and Hep G2 (human hepatocellular carcinoma cell line) obtained from American Type Culture Collection (ATCC) (Rockville, MD, U.S.A.). Hep G2 and MRC-5 cells were maintained as monolayers in Dulbecco's modified Eagle's medium (DMEM) and RPMI 1640 medium containing 10\% heat-inactivated fetal bovine serum, 100 units $/ \mathrm{mL}$ penicillin, $100 \mu \mathrm{g} / \mathrm{mL}$ streptomycin, $100 \mu \mathrm{m}$ nonessential amino acids, and $1 \mathrm{~mm}$ glutamine were prepared for cell culture.

MTT Assay for Cellular Viability Cells were seeded into 96-well plates and allowed to adhere for $24 \mathrm{~h}$ before drug were introduced. Then drugs and media were removed, and each well was treated with $100 \mu \mathrm{L}$ of $500 \mu \mathrm{g} / \mathrm{mL}$ MTT in culture medium. The plates were centrifuged at $450 \times \mathrm{g}$ for $10 \mathrm{~min}$, and supernatants were removed and replaced with $100 \mu \mathrm{L}$ of dimethyl sulfoxide (DMSO). Absorbance, as a measure of viable cell number, was read the following day in a model MA310 automated enzyme-linked immunosorbent assay (ELISA) plate reader at a wavelength of $550 \mathrm{~nm}$. $\mathrm{IC}_{50}$ values were obtained by a linear regression analysis of percent absorbance versus log drug concentration. It has been shown previously that viable cell numbers correlate with optical density as determined by the MTT assay. ${ }^{14,15)}$

$N$-(Thiazolyl)cantharidinimine (3a): $\mathrm{mp} \quad 173-175^{\circ} \mathrm{C}$ $(\mathrm{MeOH}) ;{ }^{1} \mathrm{H}-\mathrm{NMR}\left(500 \mathrm{MHz}, \mathrm{CDCl}_{3}\right): \delta$ (ppm) $1.28(\mathrm{~s}, 6 \mathrm{H}$, $\left.\mathrm{CH}_{3} \times 2\right), 1.76-1.79\left(\mathrm{~m}, 2 \mathrm{H}, \mathrm{CH}_{2}\right), 1.85-1.89\left(\mathrm{~m}, 2 \mathrm{H}, \mathrm{CH}_{2}\right), 4.74$ (t, $2 \mathrm{H}, J=2.4,2.5 \mathrm{~Hz}, \mathrm{OCH} \times 2), 7.32(\mathrm{~d}, 1 \mathrm{H}, J=3.5 \mathrm{~Hz}$, thiazolyl N-CH), 7.79 (d, 2H, $J=3.4 \mathrm{~Hz}$, thiazolyl S-CH); IR ( $\mathrm{KBr})$ 1721 (amide) $\mathrm{cm}^{-1}$; high resolution (HR)-MS (EI, $80 \mathrm{eV}$ ) Calcd for $\mathrm{C}_{13} \mathrm{H}_{14} \mathrm{~N}_{2} \mathrm{O}_{3} \mathrm{~S}$ : 278.0721. Found: 278.0721.

$\mathrm{N}$-[2-(5-Methylthiazolyl)]cantharidinimine

(3b): $\quad \mathrm{mp}$ $149-150^{\circ} \mathrm{C}(\mathrm{MeOH}) ;{ }^{1} \mathrm{H}-\mathrm{NMR}\left(500 \mathrm{MHz}, \mathrm{CDCl}_{3}\right): \delta$ (ppm) $1.26\left(\mathrm{~d}, 6 \mathrm{H}, J=5.6 \mathrm{~Hz}, \mathrm{CH}_{3} \times 2\right), 1.73-1.76\left(\mathrm{~m}, 2 \mathrm{H}, \mathrm{CH}_{2}\right)$, 1.83-1.88 (m, 2H, $\left.\mathrm{CH}_{2}\right), 2.48\left(\mathrm{~s}, 3 \mathrm{H}, \mathrm{CH}_{3}\right), 4.72$ (t, $2 \mathrm{H}, J=2.6$, $2.5 \mathrm{~Hz}, \mathrm{OCH} \times 2), 7.42(\mathrm{~s}, 1 \mathrm{H}$, thiazolyl N-CH); IR $(\mathrm{KBr})$ : 1724 (carbonyl group) $\mathrm{cm}^{-1}$; HR-MS (EI, $80 \mathrm{eV}$ ) Calcd for $\mathrm{C}_{14} \mathrm{H}_{16} \mathrm{~N}_{2} \mathrm{O}_{3} \mathrm{~S}$ : 292.0874. Found: 292.0874.

$\mathrm{N}$-(2-Benzothiazolyl)cantharidinimine (3c): $\mathrm{mp} 158-160^{\circ} \mathrm{C}$ $(\mathrm{MeOH}) ;{ }^{1} \mathrm{H}-\mathrm{NMR}\left(500 \mathrm{MHz}, \mathrm{CDCl}_{3}\right): \delta(\mathrm{ppm}) 1.31(\mathrm{~s}, 6 \mathrm{H}$, $\left.\mathrm{CH}_{3} \times 2\right)$, 1.77-1.80 (m, 2H, $\left.\mathrm{CH}_{2}\right), 1.86-1.91\left(\mathrm{~m}, 2 \mathrm{H}, \mathrm{CH}_{2}\right)$, $4.77(\mathrm{t}, 2 \mathrm{H}, J=2.6,2.6 \mathrm{~Hz}, \mathrm{OCH} \times 2), 7.42(\mathrm{t}, 1 \mathrm{H}, J=7.9,8.0 \mathrm{~Hz}$, $\mathrm{H}-5), 7.50$ (t, 1H, $J=8.2,7.5 \mathrm{~Hz}, \mathrm{H}-6), 7.89$ (d, $1 \mathrm{H}, J=8.0 \mathrm{~Hz}$, $\mathrm{H}-4), 8.12$ (d, 1H, J=8.2 Hz, H-7); IR (KBr): 1723 (carbonyl group) $\mathrm{cm}^{-1}$; HR-MS (EI, $80 \mathrm{eV}$ ) Calcd for $\mathrm{C}_{14} \mathrm{H}_{16} \mathrm{~N}_{2} \mathrm{O}_{3} \mathrm{~S}$ :
328.0882. Found: 328.0882 .

$N$-[2-(1,3,4-Thiadiazolyl)]cantharidinimine $\quad(\mathbf{3 d}): \quad \mathrm{mp}$ 201-203 ${ }^{\circ} \mathrm{C}(\mathrm{MeOH}) ;{ }^{1} \mathrm{H}-\mathrm{NMR}\left(500 \mathrm{MHz}, \mathrm{CDCl}_{3}\right): \delta$ (ppm) 1.31 (s, 6H, $\left.\mathrm{CH}_{3} \times 2\right), 1.78-1.79\left(\mathrm{~m}, 2 \mathrm{H}, \mathrm{CH}_{2}\right), 1.87-1.89(\mathrm{~m}, 2 \mathrm{H}$, $\left.\mathrm{CH}_{2}\right), 4.76(\mathrm{t}, 2 \mathrm{H}, J=2.5,2.4 \mathrm{~Hz}, \mathrm{OCHX} 2), 9.14$ (s, 1H, thiadiazolyl H); IR ( $\mathrm{KBr}$ ): 1723 (carbonyl group) $\mathrm{cm}^{-1}$, HR-MS (EI, $80 \mathrm{eV}$ ) Calcd for $\mathrm{C}_{12} \mathrm{H}_{13} \mathrm{~N}_{3} \mathrm{O}_{3} \mathrm{~S}$ : 279.0688. Found: 280.0942 .

$N$-[5-(3-Phenyl-1,2,4-thiadiazolyl)]cantharidinimine (3e): $\mathrm{mp}$ 172-174 (MeOH); ${ }^{1} \mathrm{H}-\mathrm{NMR}\left(500 \mathrm{MHz}, \mathrm{CDCl}_{3}\right): \delta$ (ppm) 1.25 (s, 6H, $\left.\mathrm{CH}_{3} \times 2\right), 1.72-1.83\left(\mathrm{~m}, 2 \mathrm{H}, \mathrm{CH}_{2}\right), 1.88-1.91(\mathrm{~m}, 2 \mathrm{H}$, $\mathrm{CH}_{2}$ ), 4.78 (t-like, $2 \mathrm{H}, \mathrm{OCH} \times 2$ ), 7.47 (t-like, 3H, overlap with H-4'),H-3', H-5', H-4'), 8.35 (dd, 2H, J=9.1, 1.2, $2.2 \mathrm{~Hz}, \mathrm{H}-2^{\prime}$, H-6'); IR (KBr) 1723 (amide) $\mathrm{cm}^{-1}$, HR-MS (EI, $80 \mathrm{eV}$ ) Calcd for $\mathrm{C}_{18} \mathrm{H}_{17} \mathrm{~N}_{3} \mathrm{O}_{3} \mathrm{~S}$ : 355.1000. Found: 355.1000 .

$\mathrm{N}$-[3-(5-Phenylpyrazolyl)]cantharidinimine (3f): $\mathrm{mp}$ 197-198 ${ }^{\circ} \mathrm{C}$; ${ }^{1} \mathrm{H}-\mathrm{NMR}\left(\mathrm{CDCl}_{3}, 500 \mathrm{MHz}\right): \delta 1.26(\mathrm{~d}, 6 \mathrm{H}, J=$ $\left.8.0 \mathrm{~Hz}, \mathrm{CH}_{3} \times 2\right), 1.76-1.77\left(\mathrm{~m}, 2 \mathrm{H}, \mathrm{CH}_{2}\right), 1.85-1.87(\mathrm{~m}, 2 \mathrm{H}$, $\left.\mathrm{CH}_{2}\right), 4.72(\mathrm{t}, 2 \mathrm{H}, J=2.6,2.4 \mathrm{~Hz}, \mathrm{OCH} \times 2), 6.98(\mathrm{~s}, 1 \mathrm{H}, \mathrm{H}-4)$, 7.37 (t-like, $1 \mathrm{H}, \mathrm{H}-4^{\prime}$ ), 7.44 (dd, 2H, J=7.7, 7.3 Hz, H-3', H-5'), 7.73 (d, 2H, J=7.5 Hz, H-2', H-6'); IR (KBr): 1706 (carbonyl group), 3284 (secondary amine) $\mathrm{cm}^{-1}$, HR-MS (EI, $80 \mathrm{eV}$ ) Calcd for $\mathrm{C}_{19} \mathrm{H}_{19} \mathrm{~N}_{3} \mathrm{O}_{3}$ : 337.1425. Found: 337.1426.

[4-(1,2,4-Triazolyl)]cantharidinimine (3g): mp $240-241^{\circ} \mathrm{C}$ $(\mathrm{MeOH}) ;{ }^{1} \mathrm{H}-\mathrm{NMR}\left(500 \mathrm{MHz}, \mathrm{CDCl}_{3}\right): \delta(\mathrm{ppm}) 1.25(\mathrm{~s}, 3 \mathrm{H}$, $\left.\mathrm{CH}_{3}\right), 1.32\left(\mathrm{~s}, 3 \mathrm{H}, \mathrm{CH}_{3}\right), 1.80-1.90\left(\mathrm{~m}, 4 \mathrm{H}, \mathrm{CH}_{2} \times 2\right), 4.73(\mathrm{t}$, $2 \mathrm{H}, J=2.6,2.4 \mathrm{~Hz}, \mathrm{OCH} \times 2), 8.31$ (s, 2H, triazolyl H); IR (KBr): 1739 (carbonyl group) $\mathrm{cm}^{-1}$; HR-MS (EI, $80 \mathrm{eV}$ ) Calcd for $\mathrm{C}_{12} \mathrm{H}_{14} \mathrm{~N}_{4} \mathrm{O}_{3}: 262.1072$. Found: 262.1072 .

$\mathrm{N}$-Cantharidinimino-sulfamethazine (6h): mp $\quad 115-117^{\circ} \mathrm{C}$ $(\mathrm{MeOH}) ;{ }^{1} \mathrm{H}-\mathrm{NMR}\left(500 \mathrm{MHz}, \mathrm{CDCl}_{3}\right): \delta$ (ppm) $1.25(\mathrm{~s}, 6 \mathrm{H}$, $\left.\mathrm{CH}_{3} \times 2\right), 1.74-1.77\left(\mathrm{~m}, 2 \mathrm{H}, \mathrm{CH}_{2}\right), 1.84-1.89\left(\mathrm{~m}, 2 \mathrm{H}, \mathrm{CH}_{2}\right), 2.35$ (s, 6H, $\left.\mathrm{CH}_{3} \times 2\right), 4.69$ (t, 2H, J=2.6, 2.7 Hz, OCH $\left.\times 2\right), 6.64$ (s, $1 \mathrm{H}$, pyrimidinyl $\mathrm{H}), 7.52(\mathrm{~d}, 2 \mathrm{H}, J=8.7 \mathrm{~Hz}$, phenyl $\mathrm{H}), 8.26$ (d, $2 \mathrm{H}, J=8.6 \mathrm{~Hz}$, phenyl H); IR (KBr): 1710 (carbonyl group) $\mathrm{cm}^{-1}$

$\mathrm{N}$-Cantharidinimino-sulfisomidine (6i): mp 99-101 $(\mathrm{MeOH}) ;{ }^{1} \mathrm{H}-\mathrm{NMR}\left(500 \mathrm{MHZ}, \mathrm{CDCl}_{3}\right): \delta$ (ppm) 1.25 (d-like, $\left.6 \mathrm{H}, \mathrm{CH}_{3} \times 2\right), 1.72-1.75\left(\mathrm{~m}, 2 \mathrm{H}, \mathrm{CH}_{2}\right), 1.85-1.88\left(\mathrm{~m}, 2 \mathrm{H}, \mathrm{CH}_{2}\right)$, $2.56\left(\mathrm{~s}, 3 \mathrm{H}, \mathrm{CH}_{3}\right), 2.68\left(\mathrm{~s}, 3 \mathrm{H}, \mathrm{CH}_{3}\right), 4.69$ (t-like, $\left.2 \mathrm{H}, \mathrm{OCH} \times 2\right)$, $6.96(\mathrm{~s}, 1 \mathrm{H}$, pyrimidinyl $\mathrm{H}), 7.56(\mathrm{~d}, 2 \mathrm{H}, J=8.2 \mathrm{~Hz}$, phenyl $\mathrm{H})$, 8.06 (d, $2 \mathrm{H}, J=8.4 \mathrm{~Hz}$, phenyl H); IR (KBr): $1710 \mathrm{~cm}^{-1}$.

$N$-(2-Pyridyl)cantharidinimine (8j): mp 159-161 (MeOH); ${ }^{1} \mathrm{H}-\mathrm{NMR}\left(500 \mathrm{MHz}, \mathrm{CDCl}_{3}\right): \delta(\mathrm{ppm}) 1.27\left(\mathrm{~s}, 6 \mathrm{H}, \mathrm{CH}_{3} \times 2\right)$, 1.74-1.76 (m, 2H, $\left.\mathrm{CH}_{2}\right), 1.85-1.89\left(\mathrm{~m}, 2 \mathrm{H}, \mathrm{CH}_{2}\right), 4.73$ (d, 2H, $J=1.8 \mathrm{~Hz}, \mathrm{OCH} \times 2), 7.33-7.36(\mathrm{dd}, 2 \mathrm{H}, J=7.6,5.14,2.97 \mathrm{~Hz}$, H-3, H-5), 7.83-7.86 (t, 1H, J=7.7,7.7 Hz, H-4), 8.64-8.65 (d, $1 \mathrm{H}, J=3.9 \mathrm{~Hz}, \mathrm{H}-6)$; IR (KBr): $1713(\mathrm{C}=\mathrm{O}) \mathrm{cm}^{-1}$; MS m/z (rel., int.); $272[\mathrm{M}]^{+},(5), 203$ (100).

$\mathrm{N}$-(5-Methyl-2-pyridyl)cantharidinimine (8k): mp 185-187 $(\mathrm{MeOH}) ;{ }^{1} \mathrm{H}-\mathrm{NMR}\left(500 \mathrm{MHz}, \mathrm{CDCl}_{3}\right): \delta(\mathrm{ppm}) 1.26(\mathrm{~s}, 6 \mathrm{H}$, $\left.\mathrm{CH}_{3} \times 2\right), 1.73-1.75\left(\mathrm{~m}, 2 \mathrm{H}, \mathrm{CH}_{2}\right), 1.84-1.87\left(\mathrm{~m}, 2 \mathrm{H}, \mathrm{CH}_{2}\right)$, $2.38\left(\mathrm{~s}, 3 \mathrm{H}, \mathrm{CH}_{3}-5\right), 4.72(\mathrm{~s}, 2 \mathrm{H}, \mathrm{OCH} \times 2), 7.21-7.23(\mathrm{~d}, 1 \mathrm{H}$, $J=8.0 \mathrm{~Hz}, \mathrm{H}-3), 7.64-7.66$ (d, $1 \mathrm{H}, J=8.0 \mathrm{~Hz}, \mathrm{H}-4), 8.45$ (s, 1H, H-6); IR (KBr): $1709(\mathrm{C}=\mathrm{O}) \mathrm{cm}^{-1}$; MS m/z (rel., int.) 286 $[\mathrm{M}]^{+}(5), 217$ (100).

$\mathrm{N}$-(3-Amino-2-pyridyl)cantharidinimine (81): mp 281-283 $(\mathrm{MeOH}) ;{ }^{1} \mathrm{H}-\mathrm{NMR}\left(500 \mathrm{MHz}, \mathrm{CDCl}_{3}\right): \delta$ (ppm) 1.29 (s, 6H, $\left.\mathrm{CH}_{3} \times 2\right), 1.75-1.79\left(\mathrm{~m}, 2 \mathrm{H}, \mathrm{CH}_{2}\right), 1.88-1.92\left(\mathrm{~m}, 2 \mathrm{H}, \mathrm{CH}_{2}\right), 4.71$ (s, 2H, OCH $\times 2), 7.14-7.15(\mathrm{~d}, 1 \mathrm{H}, J=8.0 \mathrm{~Hz}, \mathrm{H}-4), 7.19-7.21$ (m, 1H, H-5), 7.99 (s, 1H, H-6); IR (KBr): 1709 (C=O) cm $\mathrm{cm}^{-1}$. MS m/z (rel., int.) $287[\mathrm{M}]^{+}$(13), 217 (100). 
$\mathrm{N}$-(3-Amino-4-pyridyl)cantharidinimine (8m): mp 235-238 $(\mathrm{MeOH}) ;{ }^{1} \mathrm{H}-\mathrm{NMR}\left(500 \mathrm{MHz}, \mathrm{CDCl}_{3}\right): \delta$ (ppm) $1.28(\mathrm{~s}, 6 \mathrm{H}$, $\left.\mathrm{CH}_{3} \times 2\right), 1.76-1.78\left(\mathrm{~m}, 2 \mathrm{H}, \mathrm{CH}_{2}\right), 1.87-1.91\left(\mathrm{~m}, 2 \mathrm{H}, \mathrm{CH}_{2}\right), 3.97$ (bs, $\left.2 \mathrm{H}, \mathrm{NH}_{2}-3\right), 4.69-4.70(\mathrm{t}, 2 \mathrm{H}, J=2.5,2.3 \mathrm{~Hz}, \mathrm{OCH} \times 2$ ), 6.95(s, 1H, H-5), 8.06-8.07 (d, 1H, J=4.7 Hz, H-6), 8.24 (s, $1 \mathrm{H}, \mathrm{H}-2)$; IR $(\mathrm{KBr}): 1713(\mathrm{C}=\mathrm{O}) \mathrm{cm}^{-1}$. MS m/z (rel., int.): 287 $\left[\mathrm{M}^{+}(13), 170\right.$ (100).

$N$-(4-Amino-3-pyridyl)cantharidinimine (8n): mp 273-276 $(\mathrm{MeOH}) ;{ }^{1} \mathrm{H}-\mathrm{NMR}\left(500 \mathrm{MHz}, \mathrm{CDCl}_{3}\right): \delta$ (ppm) $1.29(\mathrm{~s}, 6 \mathrm{H}$, $\left.\mathrm{CH}_{3} \times 2\right), 1.76-1.78\left(\mathrm{~m}, 2 \mathrm{H}, \mathrm{CH}_{2}\right), 1.89-1.92\left(\mathrm{~m}, 2 \mathrm{H}, \mathrm{CH}_{2}\right)$, 4.51 (bs, $\left.2 \mathrm{H}, \mathrm{NH}_{2}-4\right), 4.69$ (s, 2H, OCH $\left.\times 2\right), 6.63-6.64(\mathrm{~d}, 1 \mathrm{H}$, $J=5.6 \mathrm{~Hz}, \mathrm{H}-5), 8.04$ (s, $1 \mathrm{H}, \mathrm{H}-2), 8.21-8.30$ (d, $1 \mathrm{H}, J=5.6 \mathrm{~Hz}$, $\mathrm{H}-6)$; IR ( $\mathrm{KBr})$ : $1713(\mathrm{C}=\mathrm{O}) \mathrm{cm}^{-1}$. MS m/z (rel., int.): 287 $[\mathrm{M}]^{+}(100), 218(75)$.

N-(5-Bromo-2-pyridyl)cantharidinimine (8o): mp 193-194 $(\mathrm{MeOH}) ;{ }^{1} \mathrm{H}-\mathrm{NMR}\left(500 \mathrm{MHz}, \mathrm{CDCl}_{3}\right): \delta$ (ppm) $1.26(\mathrm{~s}, 6 \mathrm{H}$, $\left.\mathrm{CH}_{3} \times 2\right)$, $1.74-1.76\left(\mathrm{~m}, 2 \mathrm{H}, \mathrm{CH}_{2}\right), 1.84-1.87\left(\mathrm{~m}, 2 \mathrm{H}, \mathrm{CH}_{2}\right)$, 4.71-4.72 (d, 2H, J=2.41 Hz, OCH $\times 2), 7.25-7.26(\mathrm{t}, 1 \mathrm{H}$, H-3), 7.95-7.97 (q, 1H, J=2.3, 6.1, 2.4 Hz, H-4), 8.69 (d, 1H, $J=2.2 \mathrm{~Hz}, \mathrm{H}-6)$; IR (KBr): $1710(\mathrm{C}=\mathrm{O}) \mathrm{cm}^{-1}$; MS $\mathrm{m} / \mathrm{z}$ (rel., int.): $350\left[\mathrm{M}^{+}\right.$(2), 281 (100), 283 (97).

$\mathrm{N}$-(4-Pyridyl)cantharidinimine (8p): mp 139-140 (MeOH); ${ }^{1} \mathrm{H}-\mathrm{NMR}\left(500 \mathrm{MHz}, \mathrm{CDCl}_{3}\right): \delta(\mathrm{ppm}) 1.26\left(\mathrm{~s}, 6 \mathrm{H}, \mathrm{CH}_{3} \times 2\right)$, 1.75-1.77 (m, 2H, $\left.\mathrm{CH}_{2}\right), 1.85-1.88\left(\mathrm{~m}, 2 \mathrm{H}, \mathrm{CH}_{2}\right), 4.69-4.70(\mathrm{t}$, $2 \mathrm{H}, J=2.7,2.61 \mathrm{~Hz}, \mathrm{OCH} \times 2), 7.46-7.47(\mathrm{~d}, 2 \mathrm{H}, J=6.2 \mathrm{~Hz}, \mathrm{H}-3$, $\mathrm{H}-5), 8.71-8.73(\mathrm{~d}, 2 \mathrm{H}, J=5.5 \mathrm{~Hz}, \mathrm{H}-2, \mathrm{H}-6)$; IR (KBr): 1717 $(\mathrm{C}=\mathrm{O}) \mathrm{cm}^{-1}$; MS m/z (rel., int.): $272[\mathrm{M}]^{+}$(38), 203 (100), 204 (95).

$N$-(4,6-Dimethyl-2-pyridyl)cantharidinimine $\quad(\mathbf{8 q}): \quad \mathrm{mp}$ 185-186 (MeOH); ${ }^{1} \mathrm{H}-\mathrm{NMR}\left(500 \mathrm{MHz}, \mathrm{CDCl}_{3}\right): \delta(\mathrm{ppm}) 1.26$ (s, 6H, $\left.\mathrm{CH}_{3} \times 2\right), 1.73-1.75\left(\mathrm{~m}, 2 \mathrm{H}, \mathrm{CH}_{2}\right), 1.85-1.87(\mathrm{~m}, 2 \mathrm{H}$, $\left.\mathrm{CH}_{2}\right), 2.35$ (s, 3H, $\left.\mathrm{CH}_{3}-6\right), 2.53\left(\mathrm{~s}, 3 \mathrm{H}, \mathrm{CH}_{3}-4\right), 4.71-4.72(\mathrm{t}$, $2 \mathrm{H}, J=2.9,2.6 \mathrm{~Hz}, \mathrm{OCH} \times 2), 6.9(\mathrm{~s}, 1 \mathrm{H}, \mathrm{H}-5), 7.03$ (s, 1H, $\mathrm{H}-3)$; IR (KBr): 1707 (carbonyl group) $\mathrm{cm}^{-1}$; MS $\mathrm{m} / \mathrm{z}$ (rel., int.) $300[\mathrm{M}]^{+}(12), 231(100)$.

$N$-(5-Hydroxy-6-pyridyl)cantharidinimine (8r): mp 289-291 $(\mathrm{MeOH}) ;{ }^{1} \mathrm{H}-\mathrm{NMR}\left(500 \mathrm{MHz}, \mathrm{CDCl}_{3}\right): \delta$ (ppm) 1.25 (s, 6H,
$\left.\mathrm{CH}_{3} \times 2\right), 1.71-1.73\left(\mathrm{~m}, 2 \mathrm{H}, \mathrm{CH}_{2}\right), 1.83-1.85\left(\mathrm{~m}, 2 \mathrm{H}, \mathrm{CH}_{2}\right)$, $4.69-4.70 \quad(\mathrm{t}, \quad 2 \mathrm{H}, \quad J=2.7,2.7 \mathrm{~Hz}, \quad \mathrm{OCH} \times 2), 7.21-7.23 \quad(\mathrm{t}$, $1 \mathrm{H}, J=4.5,4.5 \mathrm{~Hz}, \mathrm{H}-5), 7.35-7.37(\mathrm{~d}, 1 \mathrm{H}, J=6.9 \mathrm{~Hz}, \mathrm{H}-4)$, 8.10-8.12(d, 1H, J=3.2 Hz, H-6); IR (KBr): $1712(\mathrm{C}=\mathrm{O}) \mathrm{cm}^{-1}$; MS m/z (rel., int.): $288[\mathrm{M}]^{+}$(18), 219 (100).

Acknowledgments We thank Ms. Shwu-Huey Wang for help with mass and NMR spectra and data calculation.

\section{References}

1) Zhang S., Acta Pharmacol. Sin., 16, 784-786 (1981).

2) Walter W. G., J. Pharmacol. Sci., 78, 69-70 (1989).

3) Wei T. E. J-G., Lin, P-Y., Hsu F-L., Chiang H-C., Anticancer Res., 17, 2095-2098 (1997).

4) Wang G. S., J. Ethnopharmacol., 26, 147-162 (1989).

5) Fillmore K., Bagatell K., Dugan B. S., J. Invest. Dermatol., 47, 606-611 (1966).

6) Yell J. A., Burge S. M., Dean D., Br. J. Dermatol., 131, 40-47 (1994).

7) Decker R. H., J. Invest. Dermatol., 42, 465-471 (1963).

8) Twentyman P. R., Luscombe M., Br. J. Cancer, 56, 279-285 (1987).

9) Fogh J., Fogh J. M., Orfeo T., J. Natl. Cancer Inst., 59, 221-226 (1977).

10) Lin P.-Y., Shi S.-J., Hsu F.-L., Chen C.-F., J. Chin. Chem. Soc., 45, 323-326 (1998).

11) Lin P.-Y., Shi S.-J., Hsu H.-L., Chen H.-F., Lin C.-C., Liu P.-C., Wang L.-F., Bioorg. Chem., 28, 266-272 (2000).

12) Yeh C.-B., Hwang J.-M., Su C.-J., Chou M.-C., Eur. J. Med. Chem. Chem, 45, 3981-3985 (2010).

13) Ogawa T., Nozaki M., Matsui M., Tetrahedron, 36, 2641-2648 (1980).

14) van de Loosdrecht A.-A., Beelen R.-H., Ossenkoppele G.-J., Broekhoven M.-G., Langenhuijsen M.-M., J. Immunol. Methods, 174, 311-320 (1994).

15) Foongladda S., Roengsanthia D. Arjrattanakool, W., Chuchottwworn, C., Chauprasert, A., Franzblau, S-G, Int. J. Tuberc. Lung Dis., 6, 1118-1122 (2002).

16) Wang S., Wu X., Tan M., Gong J., Tan W., Bian B., Chen M., Wang Y., J. Ethnopharmacol., 140, 33-45 (2012). 